\section{Big is Beautiful: Science (and Art) in Wood Microscopy Nigel Chaffey \\ Swedish University of Agricultural Sciences, Umeå, Sweden}

\section{Abstract}

Notwithstanding the importance of trees, considerable technical problems have severely restricted study of the fine structure of the vascular cambium and the wood and bark that it produces. And despite the advent of immunocytochemical techniques, it is only in the last few years that the role of the cytoskeleton in the process of secondary vascular differentiation has been studied. Yet, for those who persist with this intractable material, the experience can be rewarding. This article discusses the difficulties involved in microscopy of this material, and offers some solutions.

\section{Introduction}

If you were told of a plant where a single cell type could give rise to 13 different cell types; or in which cells up to several millimeters long could divide perfectly for many thousands of years; or whose cells contained some of the most interesting microtubule arrays yet seen in the plant world, you might not believe it. Or, if you believed it, you would assume that it was bound to have been intensively studied already. Well, such a plant does exist, the tree. Yet its vascular cambium, which performs these wonderful feats, has largely been left untouched from a cell biological point of view. This is both surprising and disappointing, because trees are as important today as they have been for many hundreds of years, e.g., in providing wood for building or wood-pulp for paper manufacture (and notwithstanding their role in removing the excesses of $\mathrm{CO}_{2}$ liberated by the burning of fossil fuel reserves, and hence in stabilising climatic changes...). Further, given the current high level of interest in 'green issues', trees are being looked at anew as an environmentally-friendly renewable source of energy. Or, to put all this another way, TREES ARE VERY IMPORTANT.

Their importance derives largely from the activity of the vascular cambium, a sheath of tissue which encircles the stem or root, which by repeated cell division not only maintains itself but also gives rise to secondary xylem cells ('wood') to its inside and secondary phloem ('bark') to the outside. Phloem is the major long-distance transport pathway for sugar. e.g., from leaves to roots and other non-photosynthetic regions of the plant. Xylem contains vessels, the main conduits for long-distance water transport from roots to leaves. The formation of the secondary vascular tissues secondary xylem and secondary phloem - is termed secondary growth (or secondary thickening) and is largely responsible for the growth in diameter of trees. The developmental continuum of secondary phloem, cambium, and secondary xylem is termed the secondary vascular system (SVS).

In view of the importance of trees and the abundance of modern techniques for study of cell biology, why has the SVS received so little attention from developmental biologists in recent years? There are two main reasons: one, the SVS, particularly the cambium, is perceived as being difficult to study, and two, secondary growth is rarely taught to the same degree as other aspects of plant growth and development, so that it never receives the publicity it deserves.

I have an interest in the cytoskeleton in the cambium, and its role in cell differentiation within the SVS of hardwood (angiosperm) trees (Chaffey et al., 1996, 1997a,b,c). In order to study this cell component, using indirect immunofluorescence microscopy (IIF), I have had to come to terms with the idiosyncrasies of the SVS in developing techniques that would allow me to unlock some of its secrets. This article has been written to show that the SVS of trees can be studied, and to encourage others to study this fascinating developmental system.

\section{The problems...}

of relevance to any technique of study:

1) Cells of the cambium are:

a. thin-walled,

b. highly vacuolate, and,

c. sandwiched between much thicker-walled xylem and phloem cells
2) The SVS is often quite deeply embedded within the stem (or root).

3) Cambium contains two distinct cell types: axially-elongated fusiform cells, and cuboid ray cells, which eventually become elongated in the radial plane of the stem (or root).

4) The SVS contains many types of cells which are at different physiological states/stages of differentiation.

5) When the cambium is active in cell production, it tears easily during sampling.

6) Sections of the SVS adhere with difficulty to glass microscope slides.

7) The SVS is 3-dimensional.

...of relevance particularly to immunocytochemical studies:

8) The SVS contains many compounds which fluoresce naturally

9) Cell preservation is compromised by the low concentrations of fixative that can be used

10) There is difficulty in finding a suitable embedding medium.

Overcoming the 'problems'...

1) Without a doubt, these features of the cambium make it difficult to work with, but quite stunning images can be obtained (see Farrar and Evert 1997). Although, it is essential to try a range of fixation procedures to find what suits your material, fairly standard mixtures of formaldehyde and glutaraldehyde in the 'usual' range of buffers all seem suitable. And, on the 'plus' side, the very robustness of wood cells means that processing them for scanning electron microscopy can be as simple as air-drying and metal coating (e.g., Chaffey et al., 1996).

2) The location of the SVS is a major hindrance to its ready removal, a problem which is exacerbated for those who work on roots! However. as long as one works reasonably quickly (but safely!) - with hack saw and razor blade (for saplings) (Chaffey et al., 1997c), or with hammer, chisel and razor blade (for more mature specimens) (Barnett, 1992) - samples can be removed.

3) The differences between the two cell types in the cambium - and indeed in the SVS generally - potentially demand different processing strategies, If considered separately. However, the distribution of cell types is often unpredictable, so any sample contains a mixture of both. Consequently, the processing requirements of the long fusiform cells take precedence, and the key to successful preparation is to THINK BIG! During my apprenticeship in transmission electron microscopy (TEM), I was taught to have cubes of tissue with sides no greater than $1 \mathrm{~mm}$. That strategy is entirely inappropriate to the SVS where individual cells can be up to $3 \mathrm{~mm}$ long. Figure 1 illustrates a recommended sampling technique for saplings. The essential point is to excise and fix large slivers of material but with a thickness of approximately $1-2 \mathrm{~mm}$. That way the smallest dimension is in the range of 'traditional TEM'. This permits ready access of fixatives to cells, etc., whilst the larger dimensions ensure that entire cells are being fixed. After initial fixation, terminal portions of the slivers are removed (to eliminate damaged regions), before trimming to final block size, a more manageable $3 \mathrm{~mm} \times 3 \mathrm{~mm} \times 1-2 \mathrm{~mm}$. Even if whole cells are not present in these smaller blocks, the portions of cells that are present should be fixed and their structure faithfully preserved.

4) The sheer variety of cell types is one of the exciting features of the SVS, but another confounding factor to its successful microscopical study. Cells are at different stages of differentiation and it is (practically) impossible to find a procedure which will fix them all equally well. The fixation regime I use seems a reasonably good compromise as judged by the overall quality of the micrographs (see Chaffey et al., 1997b). Although it is worthwhile comparing results from a range of fixations, ultimately, you have to devise a protocol whose images you can believe in.

5) Tearing of the 'cambium' is a long-established marker of the spring reactivation of this tissue in temperate trees - when cambial cell walls become thin again after the period of winter dormancy, and cell division is resumed - and is known as 'bark slippage'. It occurs both in stems and roots and is a major problem in sampling the SVS. Although it is widely believed that the tearing takes place in the cambium, this is rarely the case. The rupture usually occurs within the zone of developing vascular derivatives where the cells have relatively thin walls and are undergoing radial expansion. Depending on whether phloem or xylem is made first upon resumption of cambial cell division, the cambium will either remain attached to the xylem, or be removed with the bark, respectively. Bark slippage occurs throughout the period when the cambium is active, but is not 
a feature of the dormant period (late autumn to spring). I only stress the need to be as careful as possible in excising and processing the slivers.

6) It is well-known that sections of wood are difficult to stick to slides (e.g., O'Brien and McCully, 1981). With sections in Steedman's wax and butylmethyimethacrylate (BMM), I have had most success using Mayer's egg albumen (Chaffey et al., 1996, 1997c). Although I have not tried gelatin (suggested by O'Brien and McCully, 1981), neither poly-L-lysine coating nor silanization of slides worked for me.

7) Of course, everything is 3-dimensional, but it is stressed that the SVS is an intimate association of axial cells derived from fusiform cambial cells (long cell axes parallel to long axis of organ) such as vessel elements, fibres, and sieve elements, and radial elements derived from ray cambial cells (long cell axes at right angles to long axis of organ), and the xylem and phloem ray cells. Therefore, to appreciate fully the inter-relations between the component cells of the SVS, it is essential to study the system in all three of the main planes, necessitating the cutting of transverse (cross) sections (TS), and radial (RLS) and tangential longitudinal sections (TLS). Consequently, considerable care is required in correct orientation of blocks when embedding. The major drawback of LS's relative to TS's is that the slightest 'deficiencies' in fixation always seem much more obvious. However with care - and luck! - excellent LS's can be obtained (see Farrar and Evert, 1997). And LS's are essential for interpretation of the cytoskeletal arrangements in the SVS, since a TS only reveals the thin parietal layer of cytoplasm in each cell. A useful tip for achieving good RLS's is to ensure that slivers are excised from the diameter of the sampled organ. Although this usually means that only two slivers can be removed at any given position, this practice will save many hours of disappointment in trying to interpret 'oblique RLS's'. However, it seems that Nature not only abhors a vacuum, she's not too keen on straight lines either, and it will soon become apparent that cells rarely lie perfectly horizontally or vertically in the organ. Thus. cells will appear to weave in and out of the plane of section, because they do! Another source of confusing images is that individual cells of the SVS are usually different lengths and frequently arranged in a non-storied arrangement. Non-storied refers to the cambium wherein the component cells overlap laterally so that they terminate at different levels in the transverse plane. Since there appears to be more cytoplasm at the ends of the cells (same thickness of cytoplasm, but smaller cell diameter), they can easily be mistaken for a different cell type, by reference to their more-vacuolate neighbours.

8) Chlorophyll, lignin and tannin are famously fluorescent materials and can be major irritants in IIF of the SVS. Depending on the fluorochromes you are using, native fluorescence may be more or less of a problem: choose with this in mind. Chlorophyll fluorescence is not a problem in roots, a much neglected area of SVS study (Chaffey et al., 1997b). However, most people concentrate on the aerial portions where chlorophyll can be a major problem, although the wavelength of maximum emission is unlikely to interfere with FITC (fluorescein isothiocyanate), the major fluorochrome used in IIF. Most of the offending pigment is removed by the ethanol during dehydration of the tissues, but note some IIF processing procedures do not employ dehydration (e.g., Funada et al., 1997)

Both tannins and lignin are particular problems, the former tending to be sequestered in vacuoles, e.g., of phloem ray cells, the latter is an integral component of cell walls of most xylem cell types, and phloem fibres. Of course, this is one of the occasions where 3-D fluorescence microscopy comes into its own (e.g., Scanalytics Inc., 1997). However, for those of us who must persist with epifluorescence, my approach has been to quench what autofluorescence I can, with toluidine blue (Chaffey et al., 1997c), and to tolerate what I can't. Indeed, the red fluorescence of lignin under the FITC filter combination is a pleasant contrast to the green/yellow fluorescence of FITC, and useful in indicating where lignification of xylem elements is beginning (e.g., Chaffey et al., 1997a). Use of cell wall polysaccharide-digesting enzymes (e.g., Chaffey et al., 1996) may contribute to partial removal of wall-located fluorescent material (and, possibly, improve access of antibodies to antigenic sites). Other attempts to boost the 'signal : noise ratio' include the use of detergent (e.g. Chaffey et al., 1996; Funada et al., 1997) to puncture the plasmalemma to facilitate better penetration of antibodies - which is especially important in thick sections - up to $50 \mu \mathrm{m}$ thick in conifers (Funada et al., 1997) and blocking of non-specific antibody binding (e.g., Chaffey et al., 1997c). One way to avoid the problem of autofluorescence altogether, and that of fluorescence-fading, is to use immunogold localisation and silver-enhancement (e.g.,. Sharon and Spiegel, 1966), although to my mind it does not produce an image which is as aesthetically pleasing as that obtained by IIF.

Allied to the problem of removing unwanted fluorescence is that of retaining the immunofluorescence. Both lab-made $p$-phenylenediamine in glycerol (Chaffey et al., 1996), and the commercially-available anti-fade mountant, Vectashield ${ }^{\text {TM }}$ (Chaffey et al., 1997c), have been successful. With Vectashield, useful FITC fluorescence can still be seen after slides have been refrigerated for several months.

9) There is usually a compromise between 'good preservation' of structure and retention of antigenicity, and everyone has to find their own. Certainly for TEM, the problem is likely to be more acute than at the light microscope level, but faithful preservation of structure is required at all levels of investigation. There seem to be two aspects to the debate: one, how much 'conventional' preservation of structure can we 'get away with' before we have no immunostaining, and, two, how can we evaluate the effect of specific fixations, or pre-fixations, used to 'enhance' particular cell features?

For conventional preservation in TEM, it is usually necessary to include some glutaraldehyde in the fixation step(s) to retain any ultrastructure (other than in walls and nuclei). For $\| F$, formaldehyde alone may be sufficient (Chaffey et al., 1997c), although addition of some glutaraldehyde will usually improve the overall
A

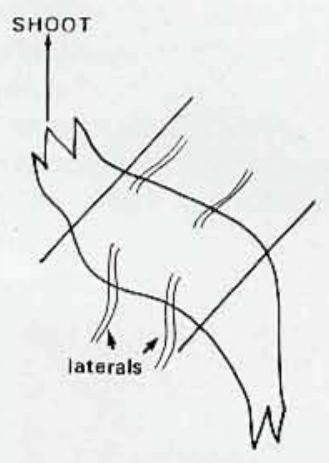

B
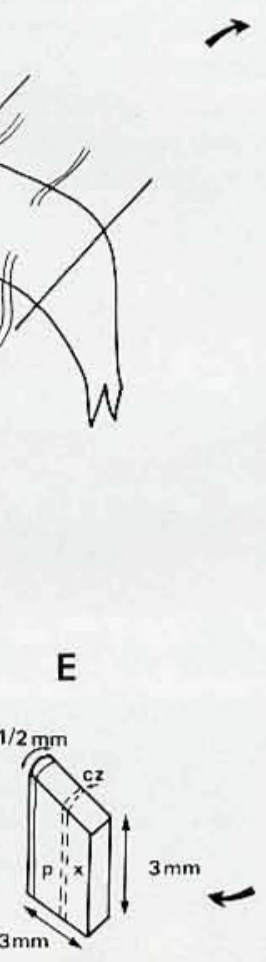

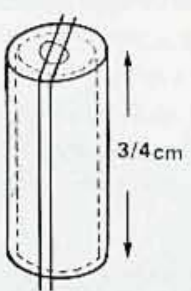

C
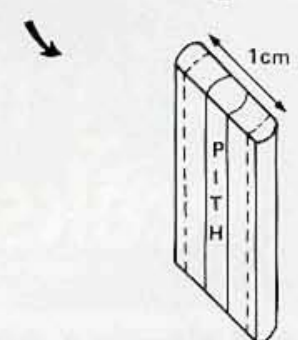

D

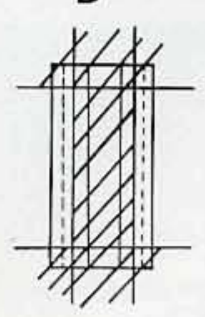

Diagrammatic representation of sampling procedure, illustrated using the taproot of Aesculus hippocastanum L. A - taproot from which samples are taken; B excised cylinder of taproot material; C - sliver removed from cylinder and ready for primary fixation (for TEM) or prefixation (for immunofluorescence microscopy); D-fixed sliver marked-out for trimming to final size; E- final trimmed block ready for post-fixation (for TEM), or overnight buffer-storage (for immunofluorescence microscopy). Abbreviations: $\mathrm{cz}$ - cambial zone; $\mathrm{p}$-phloem; $\mathrm{x}$ - secondary xylem; dashed line indicates location of $\mathrm{cz}$.

\section{Continued on Next Page}




\section{Big is Beautiful: Science (and Art) in Wood Microscopy Continued from previous page}

quality of fixation (up to $0.2 \%$ can be used in conifers (Funada et al., 1997). and in hybrid aspen by my recent experience). However, one point that is often overlooked is that the antibody may have been raised against denatured antigen, so often a relatively high level of glutaraldehyde cross-linking of protein can be tolerated by the antibody. Do not be afraid to experiment! Note, however, that glutaraldehyde can introduce additional fluorescence which may be more of a problem than that of accepting slightly inferior tissue preservation. Of course, that is not a feature of silver-enhancement or TEM, and methods do exist for removing this fluorescence, e.g., by use of $\mathrm{NaBH}_{4}$ or Schiff's reagent (Carnegie et al., 1980).

If you are concerned by the use of 'pre-fixations' such as MBS (3maleimidobenzoic acid $\mathrm{N}$-hydroxysuccinimide ester for microfilaments - Chaffey et al., 1997c), or the inclusion of DMSO (dimethyl sulphoxide for microtubules Chaffey et al. 1996; Funada et al., 1997) or 'microtubule-stabilizing buffer' (Chaffey et al., 1996), one way to check the 'reality' of the preservation is to compare the image with and without the agent (Chaffey et al., 1997c). Additionally, I would recommend a correlative approach of TEM and IIF (Chaffey et al., 1996): If two quite different preparative procedures give similar results, accept that 'consensus' view as being a reasonable approximation to reality.

10) The embedding medium must be appropriate both to the technique of study, and the information that is sought. If one is only interested in gross anatomy/histology, then wax or butyl-methylmethacrylate (BMM) is ideal (as is TEM material embedded in epoxy resin, except that the range of stains that can be used Is more limited). For ultrastructural study, Spurr's resin is Ideal (Chaffey et al., 1997b; Farrar and Evert, 1997). However, for immunogold cytochemistry, an acrylic resin such as LR White (Chaffey et al., 1997a,b) is to be preferred to epoxy resin at the TEM level (although antibody penetration/antigen accessibility may be improved in the latter by various etching methods - e.g.,. Crowley, 1997).

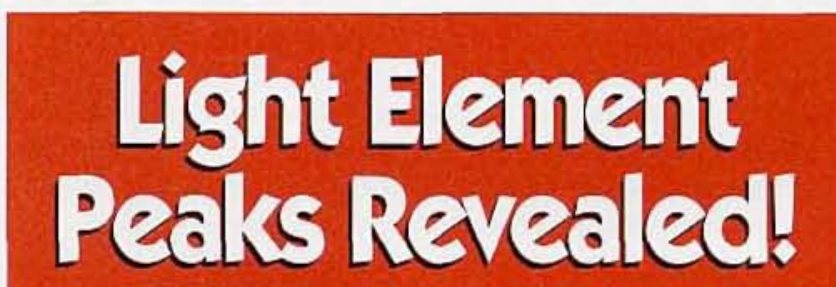

\section{Oil Film on EDX Windows Removed:}

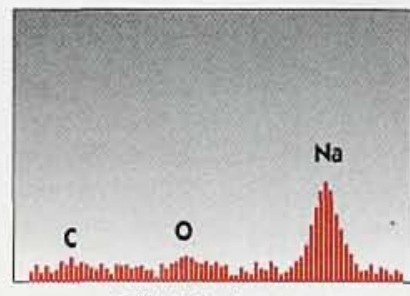

Oily Window

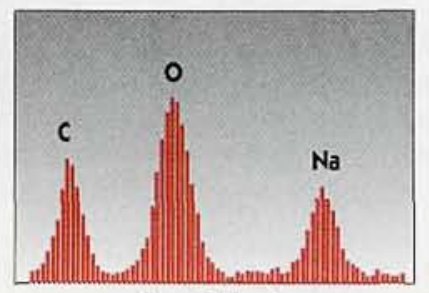

Clean Window
Oil build-up on EDX detector windows can ruin sensitivity for light element X-rays in SEMs. To stop oil condensation and keep the system clean, smart SEM users rely on the XEI Scientific SEM-CLEAN ${ }^{\top M}$ system.

Result: Consistent light element X-ray results and contaminationfree pictures. The Nitrogen purge of the inexpensive SEM-CLEAN system actively cleans your electron microscope while you're away.

\section{SEM-CLEAN ${ }^{\text {TM }}$ Stops the Oil

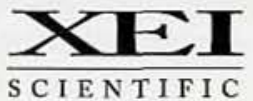

3124 Wessex Way, Redwood City, CA 94061-1348 650-369-0133 - Fax 650-363-1659

http://www.msa.microscopy.com/SM/XEI/XEIHomePage.html
For IIF of cytoskeletal proteins in very young tree material it is possible to use a low melting point wax, such as Steedman's wax (MP $37^{\circ} \mathrm{C}$ ) (Chaffey et al., 1996). However, even with small amounts of secondary thickening it is necessary to use a harder medium, such as BMM, to prevent damage to the SVS during cutting (Chaffey et al., 1997c). Although IIF study of polysaccharide epitopes using JIM antibodies is possible in LR White (Chaffey et al., 1997b), BMM gives much cleaner preparations, which I attribute to the removal of this resin prior to immunostaining.

\section{Final words}

If this article has helped to encourage others to accept the challenge of investigating the tree SVS, I will be more than happy to have played my part in helping to ensure that this fascinating area of plant cell biology does not remain neglected! The SVS is a 'challenging' system to work with. That is why it is so interesting and worthwhile, but by no means impossible. Think big, persist, good luck - and don't forget your controls for IIF (Chaffey et al., 1996)!

\section{References:}

Barnett, J.R., 1992. Reactivation of the cambium in Aesculus hippocastanum L: a transmission electron microscope study. Ann. Bot. 70; 169-177.

Carnegie. J.A., M.E. McCully, H.A. Robertson, 1980. Embedment in glycol methacrylate at low temperature allows immunofluorescent localization of a labile tissue protein. J. Hist. Cyto. 28; 308-310.

Chaffey, N.J., P.W. Barlow, J.R. Barnett, 1996. Microtubular cytoskeleton of vascular cambium and its derivatives in roots of Aesculus hippocastanum $L$ (Hippocastanaceae). In: L.A. Donaldson. B.G. Butterfield, P.A. Singh. L.J. Whitehouse, eds. Recent advances in wood anatomy. New Zealand Forest Research Institute, Rotorua, pp.171-183.

Chaffey, N.J., J.R. Barnett, P.W. Barlow, 1997a. Cortical microtubule involvement in bordered pit formation in secondary xylem vessel elements of Aescufus hippocastanum L. (Hlppocastanaceae): a correlative study using electron microscopy and indirect immunofluorescence microscopy. Protoplasma $197 ; 64-75$

Chaffey, N.J., J.R. Barnett, P.W. Barlow, 1997b. Endomembranes, cytoskeleton, and cell walls: aspects of the ultrastructure of the vascular cambium of taproots of Aesculus hippocastanum L. (Hippocastanaceae). Int. J. Plant Sci. 158; 97-109.

Chaffey, N.J., J.R. Barnett. P.W. Barlow, 1997c. Visualization of the cytoskeleton within the secondary vascular system of hardwood species. J. Microsc. $187 ; 77-84$

Crowley. H.H., 1997. Pretreating epoxy thin sections with sodium periodate prior to immunostaining. Micro. Today $97-6 ; 24-25$.

Farrar, J.J., R.F. Evert, 1997. Seasonal changes in the ultrastructure of the vascular cambium of Robinia pseudoacacia. Trees 11; 191-200.

Funada. R., H. Abe, O. Furusawa, H. Imaizumi, K Fukuzawa, J. Ontani, 1997. The orientation and localization of cortical microtubules in differentiating conifer tracheids during cell expansion. Plant Cell Physiol. 38; 210-212.

O'Brien, T.P., M. E. McCully, 1981. The study of plant structure: principles and selected methods. Termacarphi, Melbourne.

Scanalytics, Inc., 1997. High resolution 3-D fluorescence microscopy: a comparison of confocal laser scanning microscopy and a wide-field deconvolution technique. Micro. Today 97-6; 10-12.

Sharon, E., Y. Spiegel, 1996. Gold-conjugated reagents for the labelling of carbohydrate-recognition domains and glycoconjugates on nematode surfaces. J. Nematol. 28; 124-127.

We would like to thank the Royal Microscopical Society for permission to reproduce Figure 1. This figure was originally published as Figure 1 in Visualization of the cytoskeleton within the secondary vascular system of hardwood species by Chaffey, NJ, Barnett JR, Bariow PW in J. Microsc. 187, pp.77-84 (1997).

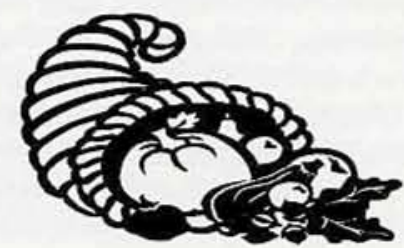




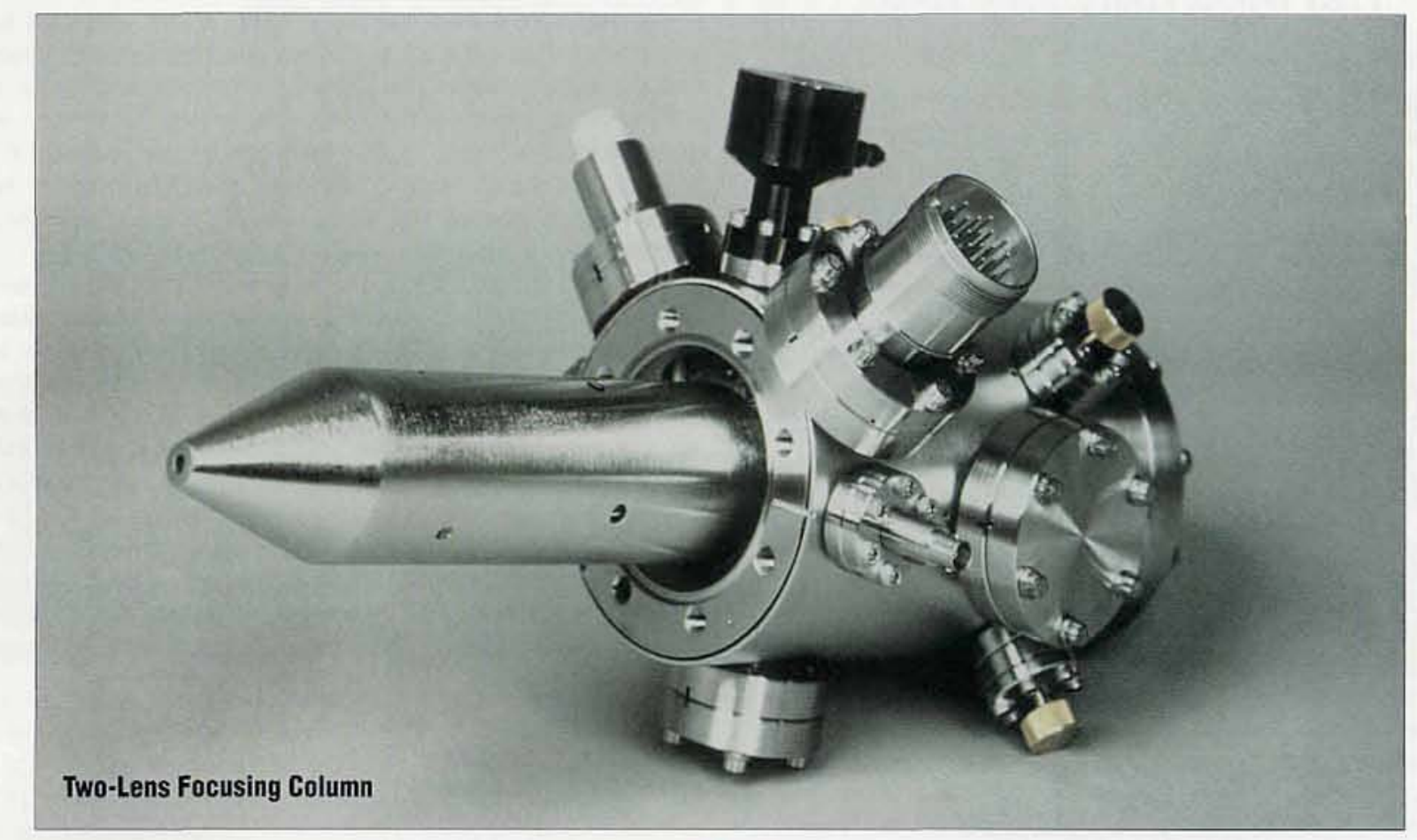

\title{
What does fi stand for?
}

Our compact, UHV, field emission columns are used by researchers worldwide. Innovative electrostatic optics and dedicated electronics allow you to integrate a high current density electron or ion column into most vacuum systems. FEI also supplies researchers with other specialized products.
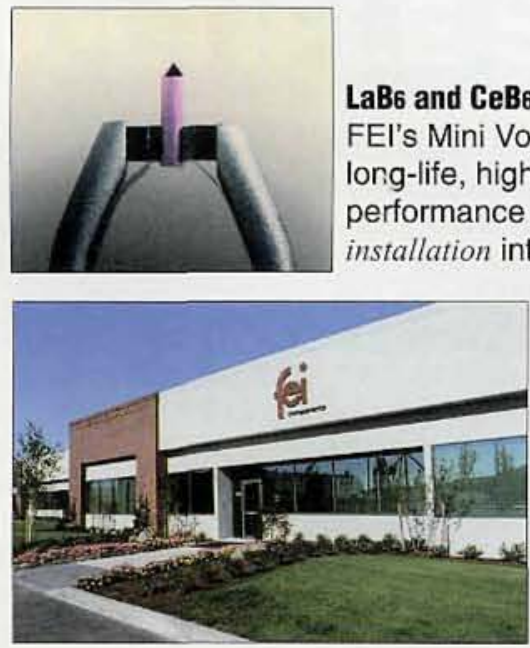

\section{LaB6 and $\mathrm{CeB}_{6}$ Cathodes}

FEl's Mini Vogel Mount, the first universally compatible long-life, high stability LaB6 cathode, provides excellent performance and the best cost-per-use value for installation into your EM systems.

\section{New Components Facilities}

Dedicated FEI Components

Group facilities enabling new

technology development through

key investments in R\&D

and manufacturing.

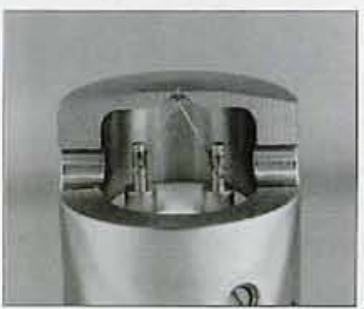

Schottky Field Emission Cathodes

FEI supplies Schottky field emitters to EM manufacturers worldwide. Schottky emission's high current intensity has established it as the preferred electron source for high resolution SEM,TEM, Auger, ESCA, EDX, and lithography.

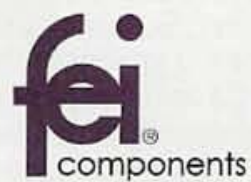

\author{
FEl Company \\ 7425 NW Evergreen Parkway \\ Hillsboro, Oregon 97124-5845 \\ (503) 844-2520 Fax (503) 640-7509 \\ E-mail <components@feico.com> \\ Subject of e-mail: "MTfei"
}

Now, when you think of FEl Components, you'll know we are the Specialists in Field Electron and Ion Technology. 